\title{
Personal Health Technology: CPN based Modeling of Coordinated Neighborhood Care Environments (Hubs) and Personal Care Device Ecosystems
}

\author{
Vijay Gehlot \\ Dept of Computing Sci and CEET \\ Villanova University \\ vijay.gehlot@ villanova.edu
}

\author{
Elliot B. Sloane \\ Dept of Computing Sci and CEET \\ Villanova University \\ elliot.sloane@villanova.edu
}

\author{
Angelo E. Thalassinidis \\ Dept of Info Sys and Technology \\ South University \\ athalassinidis@ southuniversity.edu
}

\begin{abstract}
Healthcare supported by mobile devices, or "mHealth," has rapidly emerged as a very broad ecosystem that can empower safer, more affordable, and more comfortable independent living environments and assist residents to age in place with a variety of well-understood chronic diseases. mHealth ecosystems leverage every available type of regulated medical and consumer-grade Patient Care Devices (or PCDs). mHealth technologies can also support innovative care and reimbursement models like the Patient-Centered Medical Home (PCMH) and Accountable Care Organizations (ACOs). Although consumer-grade PCDs are becoming ubiquitous, they typically do not provide a large variety of integrated system options for care coordination beyond single individuals. Understanding how to safely implement and use those devices to support heterogeneous mixes of patients, illnesses, devices, medications, and situations in neighborhood contexts is still a case-bycase challenge. By utilizing a well-formalized Colored Petri Nets (CPNs) based approach, this paper provides a proof-of-concept simulation framework for modeling and designing coordinated community care hubs.
\end{abstract}

\section{Introduction}

Healthcare supported by mobile devices, or "mHealth," has rapidly emerged as a very broad ecosystem that can empower safer, more affordable, and more comfortable independent personal healthcare living environments. mHealth technologies can also support innovative care and reimbursement models like the Patient-Centered Medical Home (PCMH) and Accountable Care Organizations (ACOs). mHealth ecosystems leverage every available type of patient Personal Care Devices (or PCDs), from smartphone-app-based weight, blood pressure, and blood glucose measurements, standalone assistive technologies like pill/medication dispensing and monitoring, all the way to fully FDAapproved medical device-based home health care $[1,2,3,4]$.

Use of lower-cost PCDs to support independent living and home health care has been stimulated by the continued escalation of US and global healthcare costs during the past decade. Regulatory roadblocks that inhibited use of apps-based PCDs, and/or constrained physician's telehealth authority and reimbursement are rapidly being eliminated in the US, Australia, and Europe [5,6,7].

In fact, Congressional FDASIA and FDA Cures legislation have encouraged FDA promulgation of relatively low-risk guidelines for apps- and softwareas-a-medical device products $[8,9,10,11]$.

The general concept of designing Community Care Coordination Hubs is not new, but prior publications have focused more on process, reimbursement, and management, rather than providing design and implementation tools like those presented in this paper [12,13,14]. In particular, [15] presents Coordination of healthcare formulated as an instance of Coordination Models defined as clientoriented coordination of cross-system transactions within a system-of-systems.

With this proposed CPN modeling tool, PCDs can be used in a heterogeneous mix of personal health devices used with, worn by, or implanted in patients/residents. The underlying technical standards can include IHE PCD, PCHA/Continua, MDPnP, HL7 v2.x and FHIR, and both the ISO/IEEE 11073.x medical device and personal health device (PHD) standards.

Currently, independent living community planners and designers who want to integrate multiple PCDs for patient home care or independent living for seniors with chronic diseases must 
individually analyze devices, understand wired and wireless system interoperability issues, design interfaces to personal or electronic medical record systems, custom-design notification and alert/alarm systems, and then try out the devices in the home environment. Each system configuration has to be treated as a pilot-effort, and troubleshooting has to be based on localized, system- and configurationspecific issues.

This is an inefficient and time-consuming process, and missed patient alerts can also introduce real health-threatening risks. Since modeling and simulation of a planned system cannot be done in advance, patient/resident care can easily suffer during the pilot testing and implementation phase, and both patient safety and medical information security can be threatened. Informed selection of interoperable and wireless medical devices has been an ongoing problem for many healthcare organizations. Medical product/device/system interoperability Conformity Assurance and/or Certification MAY eventually simplify integration of heterogeneous mixes of devices and medical record systems, but progress is only at a very early stage $[16,17,18,19,20,21]$.

Ad hoc implementation of heterogeneous medical device networks without reliable design tools can jeopardize patient lives unnecessarily, reduce system performance, waste human and fiscal capital, introduce HIPAA security risks, and expose patients to device tampering by hackers.

The realm of remote monitoring and PCD and mHealth technology use is still largely uncharacterized, particularly with respect to interoperability and reliable and secure bidirectional communication challenges. Given the current environment of rapid hardware and software innovation, the growth in affordable consumer-grade mHealth PCDs and ecosystems, health and vital sign monitoring and care is likely to continue to grow exponentially. With mHealth applications, there are numerous suites of potential devices, many of which operate on different platforms. The challenges for interoperability are numerous, complicated by the fact that there are different standards for interoperability, and conformance test tools are still needed to prove that clinical functionality can be achieved [22,23,24,25,26].

Furthermore, PCDs and mHealth products that rely on cellular technologies like smartphones and/or text messaging are presenting new challenges because they are often app-based. Using an app, a single smart device can morph into multiple separate or hybrid devices, each of which may have a different behavior and may impact the ecosystem in novel ways.
This paper proposes a generic, open-source-based starting point for a customizable modeling, simulation and testing framework for PCDs that can support relatively complex coordination of care for independent living patients/residents using a "neighborhood hub" architecture. These modeling tools allow simulation of expected ranges of safe and reliable performance, and can also be used to explore or simulate likely failure modes and potential safety or health risks due to communication system or staffing overload, errors, or other failures.

The rest of this paper is organized as follows. In section 2 we define and formalize our care coordinated health and wellness hub. In section 3 we give a brief overview of our modeling approach and notation and then provide a detailed model of the hub in section 4. We present our conclusion and future directions in section 5 .

\section{Care coordinated health and wellness hub}

We define a Care Coordinated Health and Wellness Neighborhood/Hub to consist of group of individual independent living homes or apartments (living units) loosely clustered in the neighborhood of a healthcare facility (or care coordination) hub. The hub might be a hospital, Long Term Care, Assisted Living Facility, or a Federally Qualified Health Center (FQHC), for example. Each living unit in this neighborhood may contain a variety of monitoring devices used for one or more individuals. These devices may communicate via wired or wireless (including cellular) networks. Additional coordination actors of such a hub could include family members, case managers, nurses, aides, pharmacists, physical/rehabilitation/occupational therapists, social workers, or other members of the care giving team $[27,28]$.

Finally, for the purposes of care coordination, a health information exchange and/or one or more electronic/personal medical record systems are considered an integral part of such a system of systems.

It has been noted by the Department of Health and Human Services (HHS), the ability for clinicians to monitor patients based on information, including activities of daily living measurements, captured remotely could be a key enabler for the management and control of chronic health problems and to maintain wellness for aging populations. Figure 1 depicts a typical care coordinated health and wellness neighborhood/hub per our definition, where we have explicitly identified care coordination and vigilance 
as a separate layer. This layer abstracts away clinical vigilance alerting rules are needed for various clinical care coordination categories. For example, systolic and diastolic high blood pressure alert limits (e.g., when any patient's BP exceeds either $180 \mathrm{mmHg}$ systolic or $140 \mathrm{mmHg}$ diastolic), though individual patient overrides are likely needed. There should be care coordination rules to set personal limits higher or lower based on medication and clinical situation. An automatic implementation of the rule may require the ability to fetch relevant data from an EHR, too.

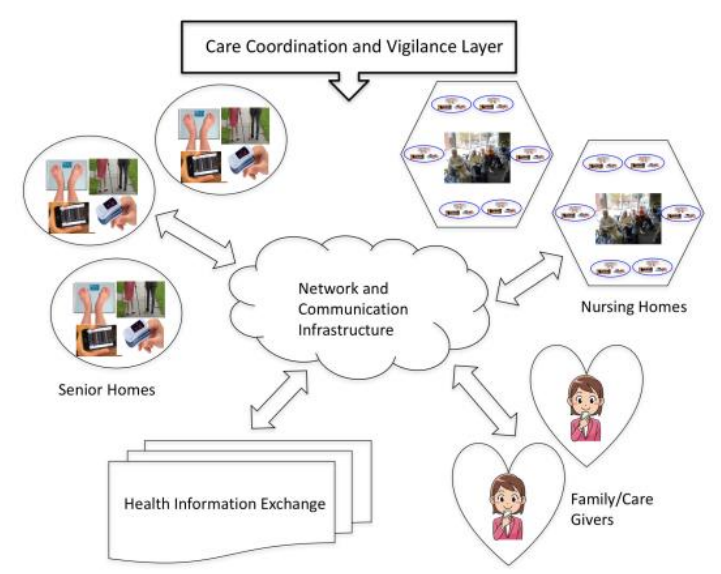

Figure 1: Components of a typical care coordinated health and wellness hub

As mentioned before, and especially from a modeling perspective, it is helpful - and realistic - to consider the hub depicted in Figure 1 above as a system of systems. Thus, a desirable modeling language/approach should at the minimum permit hierarchical and modular model building. Furthermore, the dynamicity of the hub requires a language/approach that is not only clear and explicit, but also versatile enough to support a broad variety of coordination and communication functions. Our adopted approach, which is based on Colored Petri Nets (CPNs), supports all of these features. Additionally, CPN simulation models can be integrated into live systems for testing purposes as well as for validating underlying workflows or pathways. We give a brief overview of the CPN notation next before we give details of our constructed CPN model.

\section{Colored Petri Nets}

In this section, we briefly describe the basic elements of a Petri Net (PN) and the extended formalism of Colored Petri Nets (CPNs) by means of a simple example. Petri Nets is a graphical, state-based modeling language or notation well suited for concurrent and distributed systems in which communication, synchronization, and resourcesharing play an important role. A Petri Net consists of places (depicted as circles or ovals), transitions (depicted as rectangles) and arcs (depicted as arrows) that connect a place to a transition or a transition to a place.

Places may have tokens and the firing of a transition removes tokens from its input place and adds tokens to its output place. The firing of a transition is an abstraction of the occurrence of an event and the movement of tokens describes state changes or consumption of resources. For details and tutorial introductions, see [29,30,31,32].
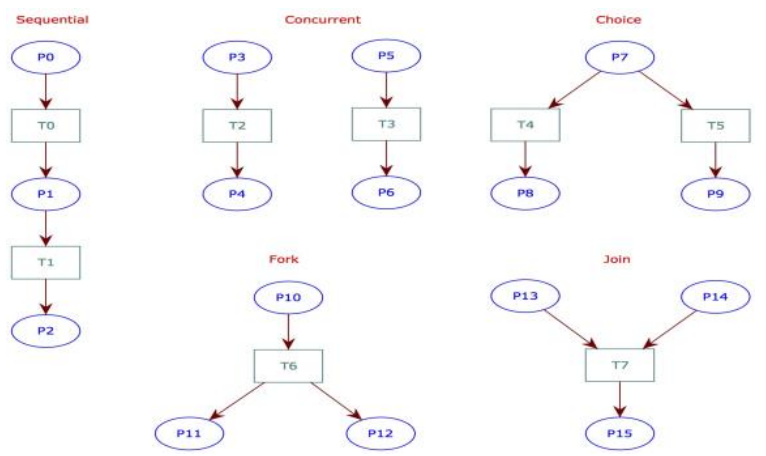

Figure 2: Petri nets configurations useful for expressing various coordination and communication of activities in healthcare settings

This state-based nature of Petri net architecture and software is an enormous virtue for critical system design, because each and every real-world action results in a clear, non-ambiguous state in the model. This characteristic allows every CPN simulation model to be rigorously verified for logic errors BEFORE running simulations that might produce erroneous results or behavior.
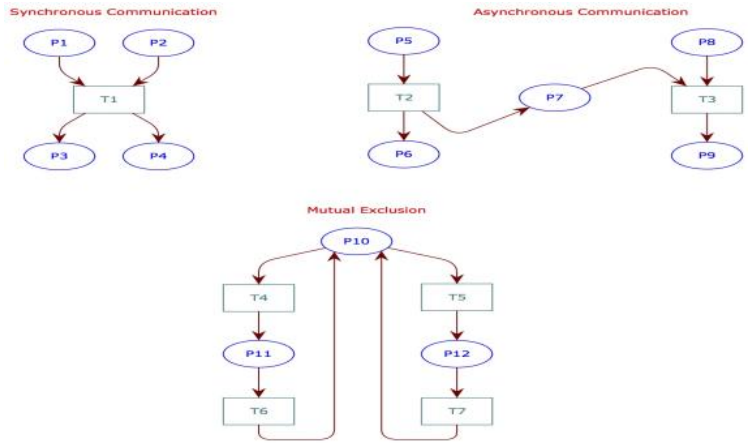

Figure 3: More Petri nets configurations useful for expressing various coordination and communication of activities in healthcare settings 
Figure 2 and Figure 3 depict some configurations of Petri nets useful for expressing various communication and coordination activities. The Sequential configuration is useful in capturing, say, the fact that a patient must see a primary care physician before consulting a specialist. The concurrent configuration captures the independence of event. For example, taking blood pressure is totally independent (thus can be concurrent) of the arrival of an ambulance. The Choice configuration is useful in capturing, say, opting for treatment $a$ vs treatment $b$.

The Join configuration in Figure 2 as well as Synchronous Communication depicted in Figure 3 are useful in modeling coordination where, say, the clinical team must come to a unanimous decision before sending the patient to the next stage of care. The Asynchronous Communication easily captures the fact of a test sample being delivered to a lab and then lab processing it asynchronously. Mutual Exclusion is useful in expressing resource sharing constraint such as a single infusion pump cannot be hooked to two different patients at the same time.

Colored Petri Nets (CPNs) are an extension of Petri Nets. CPNs extend the basic vocabulary of Petri Nets by providing support for hierarchical model constructions together with an inscription language for data representation and function definitions [30]. Specifically, under CPN, one can associate a subnet detailing the activities associated with a transition. In this case, the transition is called a Substitution Transition and the associated subnet is called a Subpage. CPN formalism permits nesting of subpages to an arbitrary depth thereby allowing system description to be captured at various levels of details. CPN models are built using a graphical software tool called CPN Tools. Furthermore, both simulation-based as well as state-space based analysis methods are supported by this tool.

\section{CPN model of the care coordinated health and wellness hub}

In this section, we give details of our constructed model as well as clarify some of the CPN specific terminology in the context of the constructed model.

CPN models facilitate relatively natural, easy, and modular hierarchical component and system construction, annotation, and testing. All components/subcomponents of the hierarchical model that we present in this section has been constructed using the CPN Tools software mentioned earlier. This software is freely available at http://cpntools.org/ and models we show here can be shared and run by any one with CPN Tools installed.

Figure 4 depicts the top-level module (or page) of our model.

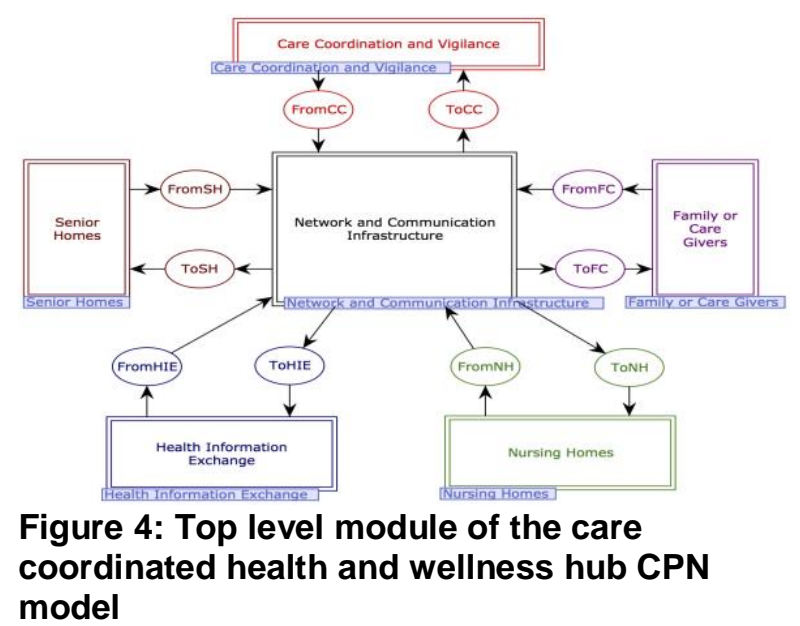

Each of the six rectangles with double border are called Substitution Transitions. Each of these Substitution Transitions represents a different page/layer/module/subsystem that becomes integrated into the entire model. For the purposes of this paper, we will use the term Layer to refer to these Substitution Transitions. In essence, each Layer can be evolved into a complex CPN subsystem if/as needed, facilitating modular design and testing. In each referenced Layer, we can assemble all needed details to support the underlying activity or subsystem.

In a CPN model, the Interfaces between each Layer are shown as circular objects in the diagram. For example, in Figure 5, the $\operatorname{To} X$ and From $X$ Interfaces will contain tokens representing any data/information packets that are flowing through the system via the network and communication fabric when the model is actually running its simulation. These Interfaces, technically called Socket Places in CPN parlance, will connect to their counterpart Port Places in the underlying Layer.

To emphasize the care coordination aspect of the model, we now separate our discussion into two parts: the hub and the coordination layer.

\subsection{Hub model components}

Figure 5 shows the subpage details for a representative Layer that we are calling IL Home (i.e., Independent Living Home). The Layer will actually consist of three substitution transitions (layers), each of which encapsulates the medical devices and activities of the underlying home. For 
our present purposes, we have shown the Layer (subpage) with three senior homes. However, there is no restriction on the number of senior homes that could belong here, nor the types of homes/locations, i.e., we can easily designate locations with names like "Diabetes Care," "Wound Care," "Diabetes and Wound Care," "Chronic Obstructive Pulmonary Care," "Congestive Heart Failure Care," "Asthma Care," "Pre-gestational Diabetes Care," etc.

For the purposes of this paper, we show the details of Senior Home 1 only in Figure 6. Each home may differ in terms of number of occupants and devices as well as associated activities. Thus, each substitution transition can be expanded to a subpage independently.

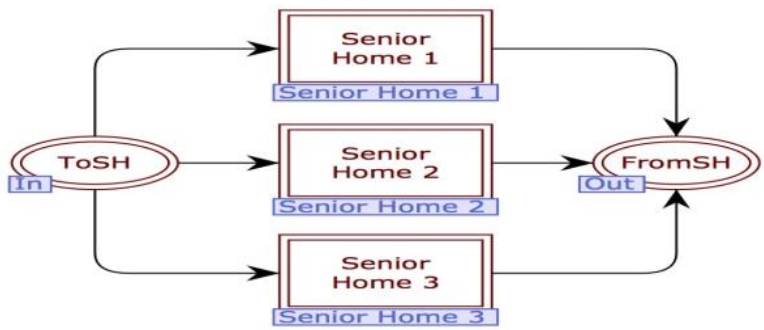

Figure 5: Sample Layer (Substitution Page) associated with "Senior Homes"

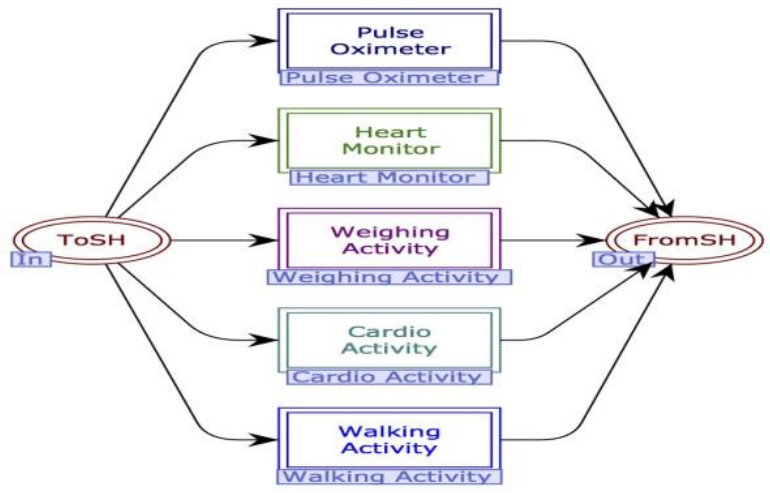

Figure 6: Subpage for Independent Living Home (Chronic Disease) with substitution transition for each medical device and activity

We assume IL Home has one occupant who has a wearable heart monitor as well as a pulse oximeter. In addition, the occupant is supposed to engage in weighing, cardio, and walking activities. Thus, from a modeling perspective, each home is a collection of devices and activities that are part of a care coordination and wellness plan. For our current purposes, we only include the details of Heart Monitor device (subpage). The associated subpage is shown in Figure 7.

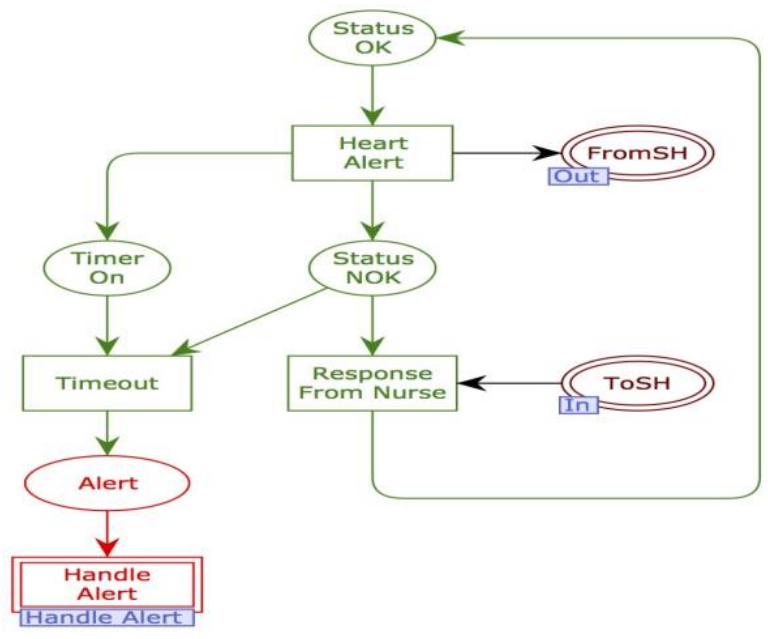
Figure 7: Subnet showing a Heart Monitor
device and associated activities

In this net, a token in place Status $O K$ indicates the fact that heart being monitored is sensed to be normal. Upon detecting abnormal heart rhythms or cardiac symptoms an alarm is generated via firing of the transition Heart Alert. The associated information/data packed is sent over the underlying network and communication fabric via the output port place FromSH and the device enter the Status $N O K$ state. If an appropriate response, per underlying care or protocol, is received via the input port place ToSH before the firing of the TimeOut transition, the device is reset to Status $O K$. However, if the timer times out, then alert handling procedures are initiated per care protocol. The substitution transition Handle Alert encapsulates those details.

As mentioned earlier, in CPN tokens may be used to carry data/information for the underlying model. CPN formalism supports both simple and compound data type construction that allows one to represent information to any level of detail.

Here we have chosen to represent a patient via a simple id consisting of a number. A device data packet is represented as a union color set consisting of either a weight (W) or Blood Pressure (BP) or Blood Oxygen (BO) or Heart Beat (HB). The BP component of the union type has two integers associated with it representing systolic and diastolic numbers. Example tokens of this type are W(220) and $\operatorname{BP}(129,80)$. This color set can be extended to include a variety of devices and their data as well as other health information. In fact, the color set construction mechanism in CPN is rich enough to be able to resent HL7 or FHIR compliant health information. 


\subsection{Care coordination model components}

The heart monitor device discussed above was assumed to have a built-in rule for an alert. However, for a care coordinated facility a more general and flexible approach is needed where custom and personalized rules may be implemented. The submodule Care Coordination and Vigilance in Figure 4, which corresponds to Care Coordination and Vigilance Layer of Figure 1, contains these details.

From a design perspective, such rules should be grouped in meaningful categories and implemented using a modular approach. For our present discussion, let us assume we have the following three categories of care coordination $(\mathrm{CC})$ rules:

1. CC Device Alert Rules for various devices.

2. CC Vigilance Alert Profiles to set patient vigilance alert profiles individually that can be used to customize device alert conditions.

3. CC Alert Escalation Rules that can be generic or customized. For example, N-Normal; CC-contact care coordinator for assessment; CF1-contact family member 1 for assessment; $\mathrm{CF} 2$-contact alternate family member for assessment; CPcontact primary care physician for assessment; DN-dispatch nurse for an unscheduled home care assessment/intervention; DU-Dispatch Uber or other transportation to transfer patient to a site for assessment; DE-Dispatch EMS service for immediate intervention. (General rules might be set for each category of care being coordinated, and individual patient/person rules might override these. For example, Do Not Resuscitate rules may require elevation to primary care physician and family coordinators, but not to the EMS.)

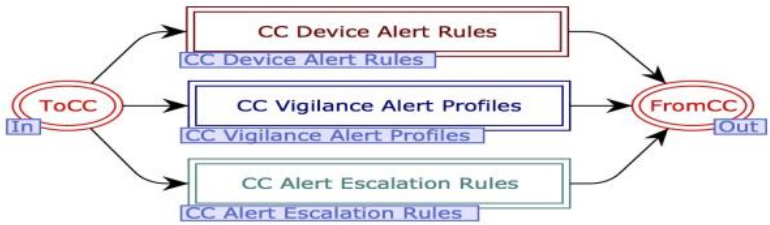

Figure 8: Model subcomponent with various care coordination rules categories

Figure 8 shows the model subpage with Layers for each of the care coordination categories described above. Let us assume we want the following rules to be part of our CC Device Alert Rules:

1. High blood pressure (hypertension): Systolic and diastolic high blood pressure alert limits (e.g., when any patient's BP exceeds either $180 \mathrm{mmHg}$ systolic or $140 \mathrm{mmHg}$ diastolic (individual patient overrides are needed, however, so that each limit can be set higher or lower based on medication and clinical situation)

2. Low blood pressure (hypotension): Systolic and diastolic high blood pressure alert limits (e.g., when any patient's BP falls below either 90 $\mathrm{mmHg}$ systolic or $60 \mathrm{mmHg}$ diastolic (individual patient overrides are needed, however, so that each limit can be set higher or lower based on medication and clinical situation) Chronic hypotension can be caused by medication, dehydration, congestive heart failure (CHF), and other conditions.

3. Low oxygen saturation (from pulse oximeter), which may relate to apnea (not breathing), or slow breathing, or obstructed breathing from asthma, or other sources. Oxygen saturations below $91 \%$ might be the general alert, but some patients with significant chronic obstructive lung disease (COPD) may hover at lower levels, especially when sleeping.)

4. Rapid continuous weight gain, steady increase of more than $4 \mathrm{lbs} /$ day for more than 3-4 days in a row for a patient with congestive heart failure (CHF) likely indicates the heart is too weak to push and pull fluids from the leg and arm extremities. Likely accompanied by lower blood pressure and lower oxygen saturation trending.

This could result in CC Devices Alert Rules being expanded to the sub Layer shown in Figure 9.

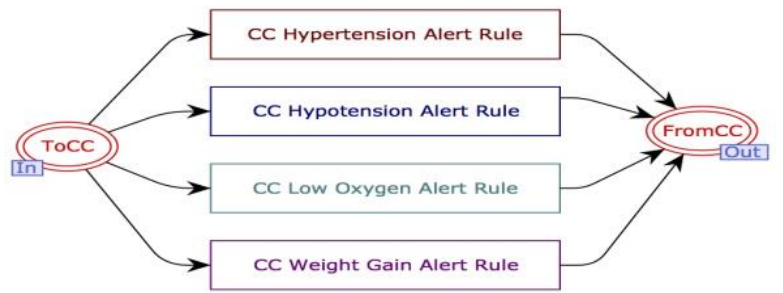

Figure 9: Model components of CC Device Alert Layer

For our present discussion, we restrict ourselves to the details of CC Hypertension Alert Rule. The sample CPN design and CPN ML programming statements shown in Figure 10 illustrates implementation of such rules.

In the net shown figure above, a token in From Device place would contain the BP readings of a patient. We assume an EMR system would contain relevant information about the patient in a patient record and we assume that this record specifies the customized upper and lower limit values for the patient. Thus, the CPN Model needs to connect to an external EMR system to fetch these limits. This is achieved using the external connection management functionality provided within the CPN Tools 
software $[33,34]$. The function getBPLimits() connects to an external database to extract the desired systolic and diastolic limit values for a given patient. These values are then used to check whether the current value from the device constitutes an alert or not. In this case, we have used the code segment (shown in blue) associated with the transition Check Hypertension Rule to compute the alert status value. An appropriate token would be placed in Status place based on the execution of the code segment when the transition fires. Finally, when the transition Handle Alert fires, an alert token is placed in Alert if there was indeed an alert, else this place would contain no token and therefore would not trigger any alerts.

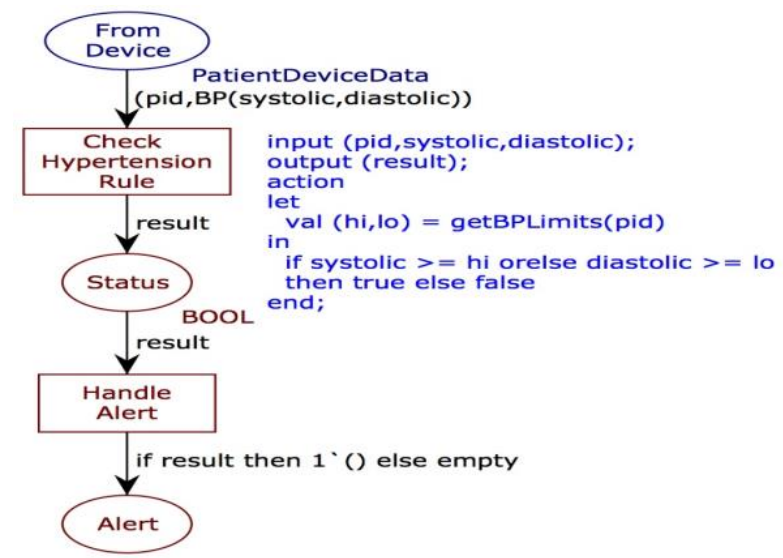

Figure 10: Implementation of CC Hypertension Alert Rule

\subsection{Model verification, validation, and simulation}

CPN Tools autonomously and automatically checks each model for broken/missing Layers, Interfaces, and/or Tokens. Every time the model is changed or extended, the new model is revalidated. A $\mathrm{CPN}$ model is executable in that it describes the state changes of a system triggered by various events (transitions). Thus, by running simulations of the CPN model, it is possible to investigate different scenarios and explore the emergent behaviors of the system under investigation.

CPN model can be run in single- or multiple-step simulation cycles, allowing point by point granular verification of triggers, pathways, and responses. For detailed performance analysis, CPN model can be instrumented with data collection monitors that log data from a simulation run and compute a variety of statistics automatically.

Once a model has been verified (i.e., performs as intended), the user may create random or statistically relevant situation-based data input streams to collect simulation run data and identify potential bottlenecks or potential failure points. For example, we can simulate potential staff overload situations when too many Alerts are occurring simultaneously, say, when asthma or cardiac complications are greatly exacerbated by multiple simultaneous days of high pollen, high temperature, high humidity. Using the CPN Tools simulations, potential staff/system overload can also be predicted based on human resource plans at a specific Care Coordination Hub. Alternately, the Care Coordination Center might use the simulation data to plan better staffing patterns, or to temporarily shift some alert-handling to a secondary or remote Care Coordination partner.

Also, "disaster simulations" can be run in the CPN Tools model, allowing "what if" failure mode planning for hurricanes or long-duration power or communication outages.

Finally, it should be noted that a simulation model should be validated before drawing any conclusions from the simulation analysis. Validation means affirming fitness for the intended purpose. In other words, if rules are entered/maintained incorrectly, or if simulated device or patient scenarios are incorrect, then the decisions and analysis will be incorrect. Therefore, such tools must have strong, competent, and well-integrated clinical leadership for safe and effective design and deployment.

\section{Conclusion and Future Work}

In this paper, we have extended prior healthrelated Color Petri Nets (CPN) modeling to support Community Care Coordination Hubs. Using CPN Tools software, we have illustrated many basic building blocks that could help design, simulate, and ultimately realize such a Hub for a diverse mix of residents and patients in Independent Living settings in nearby neighborhoods. This design and architecture assumes, of course, that the residents/patients have relatively stable, chronic conditions that can safely be monitored and managed at a distance, i.e., the patients/residents are indeed presumed to be relatively independent.

The free CPN Tools software package is uniquely valuable for this task, because:

1. The models are based on state-based decisions, and are therefore relatively easy to test and debug;

2. CPN models are modular, and CPN Tools allow assembling layered pages (modules) that can be developed and tested independently;

3. CPN models can be parameterized and CPN Tools can automatically run various configurations based on those parameters; 
4. CPN Tools automatically tests and verifies model and logic integrity as changes are made, enabling more reliable coding;

5. CPN Tools allows the developer to run singleand multiple-step simulations on an ad-hoc basis, and can aggregate performance statistics and identify potential failure or capacity overload situations in a predictive manner;

6. CPN Tools allow external process communication, which can be used to interface directly with external software and devices such as the FHIR-based LibreHealth EMR, MySQ1, or PCDs designed to use Devices on FHIR, IHE PCD, and/or medical devices or personal health devices that implement the ISO/IEEE 11073.x standards;

7. The CPN Tool model(s) can also implement internal Care Coordination Rules which can provide both global and individualized care coordination guidance;

8. The CPN Tools model can flexibly manage care coordination Alerts, routing them to one or more clinical, family, or other caregivers;

9. CPN Tools is freely licensed, which can facilitate academic, government, and commercial development and collaboration.

Our future directions include creating a university collaboratory community to share related CPN Tool models and/or modules. We would like to pilot studies with well-motivated partners like the VA, Indian Health Services, Geisinger Health Systems, or Kaiser Permanente, and/or with payors/insurers with strong care-coordination capabilities, and/or with ACOs, and or with regional Visiting Nurse Associations and/or with commercial product vendors like Apple, Fitbit, or iHealth and/or national eHealth initiatives under way in countries like South Africa.

Another opportunity for further research can emerge as the care coordinated health and wellness hub described in this paper is incrementally actualized. As described earlier, real personal and patient monitoring devices and data flow can be substituted in place of the respective CPN simulation layers. Once real user data is flowing in the system, any needed real-world adaptations can be added or fine-tuned to maintain safe and acceptable system performance.

Over time, the basic embedded decision support rules in the hub's alert and vigilance layer(s) described in Section 4.2 can be enhanced using machine learning AI tools as well, and the CPN simulation model could be used to generate synthetic data for machine learning training, as described below.
Using synthetic data for machine learning is receiving more attention recently because, like this care coordination hub, a) limited real-world data is currently available [36], and b) critical incidents, like near-collisions or accidents in self-driving car applications are quite infrequent and sparse, making real-world data collection difficult, and they may be too dangerous to repeatedly "stage" simply to gather training data $[38,38]$.

This simulated CPN care coordination hub can be used to create useful simulated data to train machine learning system that could be more flexible and adaptive than the basic vigilance layer tools described above. The CPN model can be used to create and collect simulated alert and message overload situations by steadily increasing the number of homes and alert data signals in the model while simultaneously decreasing staff responsiveness parameters. Many different combinations of alerts, such as cardiac, respiratory, or fall alerts can be intermixed, and staff response time variability and delays for handling each sort of alert can be made ever more erratic.

This simulated data could then be used to train machine learning systems in phases, so that the care coordination hub could take advantage of machine learning that is being applied in other healthcare settings. External validity of simulated datasets intended for ML training should rely on subject matter expert review to assure they represent valid healthcare scenarios, much the same way medical ML training data sets are hand-curated. This proven process was used for the American Heart Association heart arrhythmia database used to validate AI-based Automated External Defibrillators (AEDs) [46].

For example, several studies illustrate analyticsbased Remote Monitoring Systems [38, 39] as well as how these tools can be used to improve the performance of a healthcare system [40]. A number of additional articles that address real-time remote monitoring and care coordination for patients with chronic heart and lung disease are similarly useful $[41,42]$

Big data analytics that has evolved from business intelligence and decision support systems is enabling healthcare organizations to analyze an immense volume, variety and velocity of data across a wide range of healthcare networks to support evidencebased decision making and action taking [43,44]. Next we examine the benefits from and suggest strategies to implement a big data layer to the community care hub presented in section 4 utilizing the capabilities identified in [44] as well as techniques as described in [45]. 
5.1 Adding Analytical Capability for Patterns of Care Coordination

Machine-learning based analytical capabilities can identify patterns of care and discover associations from the growing body of care coordination hub data, providing a broader view for evidence-based clinical practice coordination. Using such tools, the hubs would be able to identify previously unnoticed patterns in patients related to care, coordination, and daily routines.

5.2 Additional benefits of unstructured data analytical capability

The ability to analyze unstructured data plays a pivotal role in the success of big data analytics in healthcare settings since $80 \%$ of health data is unstructured [43]. Technologies such as Speech-toText, Natural Language Processing and Sentiment Analysis can be used to both identify early warnings indicators as well as identify quality dimensions that were never identified before.

5.3 Enhanced care coordination decision support capability

Decision support capabilities emphasize the ability to produce alerts, guidance, and reports about daily healthcare and care coordination services to aid care coordinators', clinicians', and managers' decisions and actions. Using machine learning, the care coordination decision support capabilities can be more adaptive, and potentially more localized and personalized, which could result in better performance than the rule-based decision systems described in Section 2.

5.4 Enhanced care coordination predictive capability

Predictive capability is the ability to build and assess a model aimed at generating accurate predictions of new observations, [39] as an example. As [38] suggests, Machine Learning can be used for identifying medical conditions but it can also be used for less important tasks such as predicting equipment maintenance.

In closing, the authors will be glad to provide free copies of these baseline Clinical Care Coordination Hub models. The authors intend and hope that this effort can serve as catalytic event to stimulate innovation in these important areas and services.

\section{References}

[1] H. Moghimi, J. Schaffer, and N. Wickramasinghe, "Intelligent Home Risk-Based Monitoring Solutions Enable Post Acute Care Surveillance", In N. Wickramasinghe, I. Troshani, and J. Tan (eds), Contemporary Consumer Health Informatics, Healthcare Delivery in the Information Age, Springer, Cham, 2016.
[2] N. Wickramasinghe, "Pervasive Computing and Healthcare", In R. Bali, I. Troshani, S. Goldberg, and N. Wickramasinghe (eds), Pervasive Health Knowledge Management. Healthcare Delivery in the Information Age, Springer, 2013.

[3] AAMI, A Vision for Anywhere/Everywhere Healthcare, https://s3.amazonaws.com/rdcmsaami/files/production/public/FileDownloads/Summits/Hom e/HomeHealthcare.pdf, 2013, Last accessed 15 June 2018. [4] ANSI HITSP, Remote Patient Monitoring, 2008, http://hitsp.org/InteroperabilitySet_Details.aspx?MasterIS= true\&InteroperabilityId=366\&Prefix Alpha $=1 \&$ APrefix $=I S$ \&PrefixNumeric $=77$, 2008, Last accessed 15 June 2018.

[5] A. Nikolic et al., "The Use of Communication Apps by Medical Staff in the Australian Health Care System: Survey Study on Prevalence and Use", JMIR Med Inform, 6(1):e9, 2018.

[6] N. Wickramasinghe, I. Troshani, and S. Goldberg, "Adoption of Pervasive e-Health Solutions: The Need for an Appropriate Regulatory Framework", Proceedings of the AMCIS, Seattle, Washington, August 9-12, 2012, pp 1-9. [7] Veterans Health Administration, "Authority of Health Care Providers To Practice Telehealth" https://www.federalregister.gov/documents/2018/05/11/201 $\underline{\text { 8-10114/authority-of-health-care-providers-to-practice- }}$ telehealth, 2018, Last accessed 15 June 2018. [8] FDA Safety and Innovation Act (FDASIA), https://www.fda.gov/RegulatoryInformation/LawsEnforced byFDA/SignificantAmendmentstotheFDCAct/FDASIA/def ault.htm, 2012, Last accessed 15 June 2018.

[9] US Government "21st Century Cures Act", https://www.congress.gov/bill/114th-congress/housebill/34/text, 2016, Last accessed 15 June 2018.

[10] FDA Guidance: Software as a Medical Device (SAMD),

https://www.fda.gov/downloads/MedicalDevices/DeviceRe gulationandGuidance/GuidanceDocuments/UCM524904.p df, 2017, Last accessed 15 June 2018.

[11] FDA Guidance: Mobile Medical Applications, https://www.fda.gov/downloads/MedicalDevices/.../UCM2 63366.pdf, 2015, Last accessed 15 June 2018.

[12] The Atlantic, "The Big-Brother Model of Assisted Living",

https://www.theatlantic.com/health/archive/2013/01/thebig-brother-model-of-assisted-living/267035/, 2013, Last accessed 15 June 2018.

[13] RHIhub, "Community HUB Model", https://www.ruralhealthinfo.org/toolkits/services-

integration/2/care-coordination/community-hub, Last accessed 15 June 2018.

[14] ILC,

http://www.ilcuk.org.uk/index.php/news/news_posts/press release_the_care_home_of_the_future_must_become_a_co mmunity_hub, 2012, Last accessed 15 June 2018.

[15] B. P. Zeigler, "The Role of Modeling and Simulation in Coordination of Health Care", In SIMULTECH 2014 4th International Conference on Simulation and Modeling Methodologies, Technologies and Applications, 2014, pp IS-5-IS-16.

[16] E. B. Sloane and V. Gehlot, "Use of Coloured Petri Net models in planning, design, and simulation of 
intelligent wireless medical device networks for safe and flexible hospital capacity management", International Journal of Networking and Virtual Organisations (IJNVO), Vol. 4, No. 2, 2007, pp. 118-129.

[17] V. Gehlot and E.B. Sloane, "Ensuring patient safety in wireless medical device networks", IEEE Computer, 39(4):32-38, 2006.

[18] V. Gehlot and E. B. Sloane "Quality of Service (QoS) in Healthcare Applications: Colored Petri Net Simulation for Design of Heterogeneous, Multi-Vendor, Integrated, Life-Critical Wireless (802.x) Patient Care Device Networks", Proceedings of the Eleventh Americas Conference on Information Systems, Omaha, August 11th14th 2005.

[19] AAMI, FDA, and FCC, "Healthcare Technology in a Wireless World" http://s3.amazonaws.com/rdcmsaami/files/production/public/FileDownloads/Summits/2012 _Wireless_Workshop_publication.pdf, 2012, Last accessed 15 June 2018.

[20] HL7 Devices on FHIR,

http://wiki.hl7.org/index.php?title=Devices_on_FHIR, Last accessed 15 June 2018.

[21] ANSI HITSP TN-905, "Device Connectivity" http://hitsp.org/ConstructSet_Details.aspx?\&PrefixAlpha=5 \&PrefixNumeric $=905$, Last accessed 15 June 2018. [22] AAMI Medical Device Interoperability Summit, http://www.aami.org/events/eventdetail.aspx? ItemNumber $=1283$, 2012, Last accessed 15 June 2018.

[23] F. Oemig, R. D. Snelick, Foundations of Healthcare Interoperability, 2016 Springer Science+Business Media, 2016.

[24] E. B. Sloane, "Connecting Medical Devices to Health”, INCOSE 2016 Annual Meeting, http://www.omgwiki.org/MBSE/lib/exe/fetch.php?media= mbse:healthcare:s2b__ebsloane-incose16healthcareworkshop_ihe-ed.pdf, 2016, Last accessed 15 June 2018.

[25] HIMSS Innovation Center ConCert Certification Program, http://www.himssinnovationcenter.org/concerthimss, Last accessed 15 June 2018.

[26] IHE International Conformity Assessment Program, https://ihe.net/Conformity-Assessment/, Last accessed 15 June 2018.

[27] T. Widmer et al., "Improving knowledge provision for shared decision making in patient-physician relationships A multiagent organizational approach", In Proceedings of the 47th Hawaii International Conference on System Sciences, pp 646-655, 2014.

[28] S. W. Smith and R. Koppel, "Healthcare information technology's relativity problems: a typology of how patients' physical reality, clinicians' mental models, and healthcare information technology differ", Journal of the American Medical Informatics Association : JAMIA, 21(1):117-131, 2014.

[29] W. Reisig, Understanding Petri Nets, Spriger-Verlag, 2013.

[30] K. Jensen and L. M. Kristensen, Coloured Petri Nets: Modelling and Validation of Concurrent Systems, Springer-Verlag, 2009.

[31] K. Jensen and L. M. Kristensen, "Colored Petri Nets:

A Graphical Language for Formal Modeling and Validation of Concurrent Systems", Communications of The ACM , Vol. 58, No. 6, pp. 61-70, 2015.

[32] V. Gehlot and C. Nigro, "An Introduction to Systems Modeling and Simulation with Colored Petri Nets,"

Proceedings of the 2010 Winter Simulation Conference, pp. 2010, pp 104-118.

[33] G.E. Gallasch and L.M. Kristensen, "COMMS/CPN: A communication infrastructure for external communication with Design/CPN", In Third Workshop and Tutorial on Practical Use of Coloured Petri Nets and the CPN Tools, , 2001, pp 75-91.

[34] CPN Tools Documentation, Connection management functions, http://cpntools.org/2018/01/09/connectionmanagement-functions/, Last accessed 15 June 2018. [35] J. Tremblay et al., "Training Deep Networks with Synthetic Data: Bridging the Reality Gap by Domain Randomization", In IEEE/CVF Conference on Computer Vision and Pattern Recognition Workshops, 2018. DOI 10.1109/CVPRW.2018.00143

[36] T. Le, A. Baydin, R. Zinkov, and F. Wood, "Using Synthetic Data to Train Neural Networks is Model-Based Reasoning" 2017. https://arxiv.org/pdf/1703.00868.pdf, last accessed at 8/31/2018.

[37] A. Poibrenski, J. Sprenger, and C.Muller, "Towards a methodology for training with synthetic data on the example of pedestrian detection in a frame-by-frame Symantec segmentation task", In SEFAIS '18- Proceedings of the 1st International Workshop on Software Engineering for AI in Autonomous Systems. 2018.

[38] S. Ivan Lee, et al., "Remote patient monitoring: what impact can data analytics have on cost?" In Proceedings of the 4th Conference on Wireless Health (WH '13), 2013.

[39] M. Lan et al.,"WANDA: an end-to-end remote health monitoring and analytics system for heart failure patients", In Proc. of the Conference on Wireless Health, 2012.

[40] G. Guidi et al., "A multi-layer monitoring system for clinical management of Congestive Heart Failure", BMC Medical Informatics \& Decision Making. 2015.

[41] G. Guidi et al., "A Machine Learning System to Improve Heart Failure Patient Assistance", IEEE Journal of Biomedical and Health Informatics, Vol. 18, No. 6, 2014.

[42] G. Guidi e al., "Random Forest For Automatic Assessment of Heart Failure Severity", 35th Annual International Conference of the IEEE EMBS, 2013. [43] H. J. Watson, Tutorial: big data analytics: concepts, technologies, and applications. Commun. Assoc. Inf. Syst. 34 (1), 1247-1268, 2014.

[44] M. Khalifa and I. Zabani. "Utilizing health analytics in improving the performance of healthcare services: A case study on a tertiary care hospital", Journal of Infection and Public Health. Volume 9, Issue 6, pages 757-765. 2016. [45] Y. Wang, L. A. Kung, and T. A. Byrd, "Big Data analytics: Understanding its capabilities and potential benefits for healthcare organizations", Technological Forecasting and Social Change, Vol. 126, pp 3-13. 2018. [46] ECRI Institute, American Heart Association ECG Database USB,

https://www.ecri.org/components/Pages/AHA_ECG_USB. aspx, last accessed at 9/19/2018. 